\title{
A stimulus-location effect in contingency-governed, but not rule-based, discrimination learning.
}

\author{
Christina Meier, Stephen E. G. Lea \& I. P. L. McLaren \\ University of Exeter
}

Corresponding author:

\author{
Christina Meier \\ University of Exeter \\ School of Psychology \\ Exeter, United Kingdom
}

Phone: +44 1392264633

Fax: +44 1392724623

Email: cm374@exeter.ac.uk

Manuscript submitted for publication. Please do not cite or quote without permission from the first author.

(C) 2015 C. Meier, S. E. G. Lea \& I. P. L. McLaren 


\begin{abstract}
We tested pigeons' acquisition of a conditional discrimination task between coloured grating stimuli that included choosing one of two response keys, which either appeared as white keys to the left and right of the discriminative stimulus, or were replicas of the stimulus. Pigeons failed to acquire the discrimination when the response keys were white disks but succeeded when directly responding to a replica of the stimulus. These results highlight how conditioning processes shape learning in pigeons: the results can be accounted for by supposing that, when pigeons were allowed to respond directly towards the stimulus, learning was guided by classical conditioning; responding to white keys demanded instrumental learning, which impaired task acquisition for pigeons. In contrast, humans completing the same paradigm showed no differential learning success depending on whether figure or position indicated the correct key. However, only participants who could state the underlying discrimination rule acquired the task, which implies that human performance in this situation relied on the deduction and application of task rules instead of associative processes.
\end{abstract}

\title{
KEYWORDS
}

rule-based learning - contingency-governed learning - dual-system theory - humans - pigeons 
It sometimes happens that an apparently small change in the procedure of a learning experiment results in a sharp difference in speed of acquisition, despite the formal structure of the task being unchanged. One example is what Peterson and Trapold (1980) call the "differential outcomes effect" in conditional discrimination (for a review, see Goeters, Blakely \& Poling, 1992). In a conditional discrimination, different stimuli indicate which of two responses will be reinforced; the differential outcomes effect is that learning is faster if the outcomes of the two responses are different, for example if they involve different reinforcers (e.g. Peterson \& Trapold, 1980) or they lead to different locations (e.g. Sheldon, 1967). A second example is the stimulus adjacency effect reported by Beecher and Harrison (1971) and reviewed by Neill and Harrison (1987): a conditional discrimination between locations of an auditory stimulus is acquired much more rapidly when the response required for each sound is near its source, rather than near the source of the alternative sound.

Analysis of such unexpected effects frequently sheds light on the ways in which the subject's learning processes interface with the logic of the procedure. The present paper reports a further case of a minor procedural change producing a major difference in rate of acquisition, again in a conditional discrimination procedure. The phenomenon was noted, but not explored systematically, in the course of pilot work with pigeons for experiments reported by Maes, De Filippo, Inkster et al. (2015). The principal aim of the present paper was to investigate the phenomenon in a controlled experiment.

In addition, we wished to see whether the phenomenon would occur not only in pigeons but also in humans, since Maes et al. observed substantial differences between the behaviour of humans, on the one hand, and pigeons and rats, on the other hand, in their experiments. A number of recent papers have explored differences between human and animal performance in 
cognitive tasks (e.g. Maes et al., 2015; Smith, Ashby, Berg et al., 2011; Wills, Lea, Leaver et al., 2009), and the second aim of the present paper is to add to this strand of literature. Many differences between learning in humans and non-human learning can be attributed to the human tendency to formulate verbal rules to guide behaviour (Skinner, 1969). It is possible that nonhumans also formulate rules in some sense and in some situations, such as serial pattern learning (e.g. Kundey \& Fountain, 2014), but evidence that they do so in discrimination learning remains tentative (e.g. Katz, Bodily \& Wright, 2008). One example of a human/non-human difference that can be attributed to human rule-formulation is the generalization of learned behaviour to novel situations. In principle, this can be achieved either based on the perceptual similarity of the features of a familiar stimulus to the features of a novel stimulation, or by applying rules about the correct behaviour in familiar situations to the novel stimulation, and there is substantial evidence that humans use such rules under conditions where other animals do not (Erickson \& Kruschke, 1998; Shanks \& Darby, 1998; Pothos, 2005; Wills, Graham, Koh, McLaren, \& Rolland, 2011). Maes et al. (2015) showed that both pigeons and rats generalised from learned discrimination tasks to novel stimuli on the basis of similarity, whereas humans under the same conditions generalised according to rules.

In Maes et al.’s Experiment 2, pigeons and humans simultaneously learned negative and positive patterning problems in a go-left/go-right paradigm that required them to choose one of two copies of a target stimulus presented on the left or the right side of a computer screen. In unpublished pilot work for that experiment, the authors observed an unexpected difference in learning rates between pigeons trained under two slightly different conditions. The two conditions they used were as follows: in the first, a discriminative stimulus appeared at a central location; once a response had been made to it, two identical white response keys appeared to left 
and right, and responses to the left or the right key were reinforced depending on what stimulus was presented on the centre key. Since the response was made towards white response keys, we shall call this the "White Keys" condition. The second condition involved a very similar sequence of events, but instead of being white, the response keys were replicas of the discriminative stimulus, and hence we call this the "Replicas" condition. The subject's task is the same under both conditions, but in the pilot work for the experiments of Maes et al., the pigeons trained under the White Keys condition learned only very slowly, whereas those trained under the Replicas condition learned much faster.

Maes et al. (2015) went on to use the more successful of the two procedures, and did not carry out a controlled comparison between them. However, the difference in speed of learning between two such apparently similar procedures was sufficiently striking to deserve investigation. How could such a simple alteration in the visual appearance of the response display determine pigeons' success or failure to acquire a discrimination problem? The present paper reports a systematic experiment that was designed to confirm the difference between the two conditions tried by Maes et al., and suggest some interpretations of it.

Subjects were trained on go-left/go-right discriminations in which a target stimulus had to be classified based on one of its visual features. We manipulated the specific way a go-left/goright response was made: after seeing the target stimulus in the centre of a the computer display, subjects made a response either by choosing one of two neutral white response keys (White Keys condition) or by choosing one of two identical replicas of the stimulus (Replicas condition). In both cases, the images appeared in the same response locations on the left and the right side of the display. We carried out the experiment with both pigeons and humans, so far as possible in identical conditions, in order to investigate whether the difference in learning rates would occur 
in humans as well as pigeons, and, if it did not, to examine whether this species difference could be attributed to human use of rules. We also carried out a supplementary test with a second group of pigeons, in order to test a possible explanation of the effect; this test used somewhat different conditions, so that we could see whether the basic effect was robust to procedural variations.

\section{METHODS}

\section{Subjects}

24 Psychology undergraduate students (2 male), in exchange for course credit, and 14 pigeons (Columba livia) learned conditional discrimination tasks. Pigeons, obtained as discards from local fanciers, were maintained at or above $80 \%$ of their free-feeding weight by controlled feeding after experimental tests. They were kept in an indoor aviary at the University of Exeter, along with other pigeons.

Both humans and pigeons were naïve to the stimuli, though pigeons had previously been trained to peck at a white observing key presented in the centre of a black touch-sensitive display, followed by a peck at a red, blue, green or yellow coloured circle appearing in the same position, and finally, to peck at a white reward key randomly presented either to the left or to the right of the display to receive access to a food magazine. Humans received no instruction as to how to approach the task other than to "try to respond as fast as possible whilst making as few errors as possible".

All the humans and seven of the pigeons took part in the main experiment; the remaining seven pigeons were used in a supplementary test.

\section{Apparatus}


Pigeons were tested in one of eight identical $71 \times 50.5 \times 43.5 \mathrm{~cm}$ operant chambers. One of the long walls of the chamber was fitted with a $31 \times 23.5 \mathrm{~cm}$ (15") touch monitor (Model 1547L 1024x768pxl TFT monitor, CarrollTouch infrared detector, ELO Touchsystems Inc.) mounted $12 \mathrm{~cm}$ above the grid floor of the chamber. Two 2.8-Watt white houselights were mounted to either side above the screen; below the screen, mounted $4 \mathrm{~cm}$ above the chamber floor and directly below each house light, two $6 \times 5 \mathrm{~cm}$ apertures gave access to grain hoppers when solenoids were activated. The food hoppers were illuminated by a 2.8 -Watt light when activated and contained a 2:1 mixture of hemp seed and conditioner. Also mounted below the screen between the two food hoppers, a 50-Ohm loudspeaker played white noise into the box as well as indicating effective pecks to target areas with an immediate beep. The interior of the box was monitored by a video camera attached to the short wall of the chamber opposite the chamber door. The program used for the pigeon experiment was written in Microsoft Visual Basic 6 and run on a Dell PC using Whisker (Cardinal \& Aitken, 2010). Humans were tested in a soundproof, single-occupancy test room at the University of Exeter. The program used to run the human study was written in MATLAB R2008b® using the Psychtoolbox (Kleiner, Brainard, \& Pelli, 2007) add-on and run on an iMac using MATLAB $2011 \mathrm{~b}$.

\section{Procedure}

-----Figure 1 about here------

As displayed in Figure 1, trials started by displaying a circular coloured observing key, 200 pixels in diameter, in the centre of the computer screen - for half of the subjects, this circle 
was filled randomly in either red or green, for the other half, it was yellow or blue. Human participants were asked to mouse-click on it, pigeons pecked it on a touch screen. Following this action, the stimulus appeared. Again, a click or peck at the stimulus was required. Stimuli, made up as circular Gaussian patches of 200 pixels in diameter, consisted of one of four sinusoidal grating patterns in the same colour as the observing key, differing from one another in two dimensions: spatial frequency - either 2 or 12 cycles per 100 pixels - and line orientation either horizontal or vertical. One of these two visual dimensions determined the correct response: half the subjects in each condition had to classify stimuli based on the orientation of the grating pattern, and the other half based on its spatial frequency. That is, a subject might be trained to choose left when a vertical pattern was shown and choose right when the pattern was horizontal, while another subject was trained to go left when seeing a low spatial frequency and go right for stimuli with a high spatial frequency.

In the main experiment, 12 humans and 4 pigeons experienced Condition White Keys: the coloured stimulus remained in the centre after a response to it, and two white disks of 100 pixels in diameter appeared to its left and right side (at a distance of 250 pixels from the display centre to the centre of the white disk) and remained visible until a correct response was made. The remaining twelve humans and three pigeons experienced Condition Replicas: in this condition, after the response to the stimulus, it was deleted from the display centre and reappeared on both the left and right side of the display, at a distance of 250 pixels from the display centre to the centre of a replica. In both conditions, the stimulus indicated the correct response (click/peck the left or right response key). Incorrect responses had no scheduled consequences, so the trial continued until a correct response was made. Once a correct response was made, pigeons received access to a food magazine for 2.5 seconds. Humans made a single 
response; a correct response was indicated by the appearance of the word "CORRECT!" in the display centre and a gold star at the location of the correct response; an incorrect response was followed by the appearance of the word "WRONG!" in the display centre.

Pigeons completed a maximum of 20 daily sessions of 73 trials (the first trial of each session served as a "warm-up" trial; it was the same as the last trial of that session and was not included in the analyses). Acquisition of the task was considered successful if a pigeon responded correctly on at least $80 \%$ of trials for three successive sessions, and no further training was given once the pigeon reached this criterion. Pigeons that failed to reach this criterion under their original training condition within 20 sessions were tested on the alternative condition. Human participants completed 10 blocks of at least 8 trials; a block ended when a participant answered $80 \%$ or more of a sequence of eight trials correctly. At the end of the experiment, human participants were asked whether they had noticed any relationship between a stimulus and the correct response. If they were able to report the relevant discrimination rule (e.g., horizontal stimuli required choosing the right key while vertical stimuli indicated a response to the left), we assumed that they had relied on this rule when responding. If participants were unable to report a rule, we assumed that an ability to reliably choose the correct response was due to the automatic formation of stimulus-response associations.

For the supplementary test, seven pigeons were trained in both the orientationdiscrimination and the spatial-frequency-discrimination tasks under Condition Replicas as described above. Initially, the two discriminations were trained in separate sessions, but once both were being made accurately, trials using the different discriminations were included within a single session, first in blocks and ultimately completely interleaved. The colours that were presented before the stimulus and subsequently underlay each stimulus served as cues to indicate 
the currently relevant task: for example, a red or green cue colour indicated that the stimulus had to be categorised according to its spatial frequency, while a blue or yellow colour indicated categorisation according to the orientation of the stimulus.

Training continued until the pigeons reached stable performance levels at or above $80 \%$ accuracy in both tasks while switching frequently (e.g., from one trial to the next within the same test session) between the two tasks. Once criterion was reached, this procedure was continued in ten further 73-trials sessions of Condition Replicas. Three of the pigeons then proceeded with this task-switching setup in five 73-trials sessions that were identical in display to Condition White Keys described above. Then, five refresher sessions of Condition Replicas were administered to ensure a stable level of performance at or above $89 \%$ accuracy. Finally, these three pigeons completed five further sessions of 73 trials each, in which, as in Condition White Keys, response locations were indicated by white response keys, but, contrary to Condition White Keys, instead of presenting the target stimulus in the centre of the display until a response was made, it was deleted from the screen when the response keys appeared. The centre of the response display was left blank in this case, and we shall thus refer to this condition as "Blank". The other four pigeons experienced Condition Blank first and (after five refresher sessions of Condition Replicas) Condition White Keys second.

To measure performance in the main experiment, we assessed both the number of trials required to reach the success criterion and average error rates until completing the experiment. Data from the main experiment were analysed for each species separately in a multivariate ANOVA with Condition (White Keys or Replicas) and Task (Orientation Discrimination or Spatial-Frequency Discrimination) as between-subjects factors. In the supplementary test, we recorded error rates across sessions in each condition and compared performance using repeated- 
measures ANOVA with the within-subjects factor Condition (Replicas, White Keys or Blank). Where applicable, significance levels were subject to Huynh-Feldt corrections.

\section{RESULTS}

Performance by pigeons and humans in the main experiment is illustrated in Figures 2A and 2B. For pigeons, performance levels differed between Condition White Keys and Condition Replicas. Figure 3 shows that all pigeons in Condition White Keys failed to approach the success criterion within the 20 training sessions allowed (1460 trials), while those in Condition Replicas reached the criterion significantly sooner, in a mean of 608 trials ( 8 sessions; $S D=329$ trials), $F(1,3)=55.1 ; p=.005 ; \eta_{\mathrm{p}}^{2}=.95,95 \% \mathrm{CIs}=0.0, .97$; for these and subsequent analyses, pigeons that failed to reach the criterion were, conservatively, treated as though they had reached it in 1460 trials. Similarly, error rates were significantly lower in Condition Replicas $(21 \%, S D=6 \%$, versus $41 \%, S D=6 \%$, in Condition White Keys), $F(1,3)=13.3 ; p=.035 ; \eta_{\mathrm{p}}{ }^{2}=.82,95 \%$ CIs $=$ $0.0, .91$. After completion of the 20 training sessions, the pigeons that had failed task acquisition in Condition White Keys were trained under Condition Replicas, and learned the relevant discrimination task within 292 trials (4 sessions). As seen in Figure 3, they were faster to acquire the task and more accurate in Condition Replicas than they had been in Condition White Keys, though because of the limited number of subjects the difference was not significant (number of sessions to reach criterion: Wilcoxon $T=0, p=.068$ two-tailed; average error rate in final five sessions before transfer (37\%) vs. first five sessions after transfer $(22 \%)$ : Wilcoxon $T=0, p=$ .068 two-tailed). 
Two human participants, both in Condition Replicas, failed to reach the success criterion within ten test blocks. Their data were replaced by testing two additional participants. Humans showed no differences in acquiring a discrimination task between conditions, either in the number of trials to reach criterion $(F(1,20)=0.6 ; p=.47)$ or in their error rates $(F(1,20)=0.9 ; p$ $=.36)$. However, they made more errors when they had to learn the orientation-discrimination task (mean error rate: $18 \% ; S D=14 \%$ ) than when participants had to learn the spatial-frequencydiscrimination task (mean error rate: $7 \% ; S D=10 \%), F(1,20)=5.6 ; p=.028 ; \eta_{\mathrm{p}}{ }^{2}=.22,95 \%$ CIs $=0.0, .47$. This was not the case for pigeons, $F(1,3)=1.9 ; p=.27$. Although the Condition-byTask interaction did not reach statistical significance $(F(1,20)=3.0 ; p=.097)$, Figure 4 illustrates that it was primarily humans who had to learn the orientation-discrimination task in Condition Replicas who showed increased error rates compared to participants in the other conditions.

The species difference in learning depending on condition was confirmed by a significant Condition-by-Species interaction when analysing error rates in a univariate ANOVA including Species, Condition and Task as between-subjects factors, $F(1,23)=5.2 ; p=.032 ; \eta_{\mathrm{p}}{ }^{2}=.19,95 \%$ CIs $=0.0, .43$ : again, the pigeons' performance was heavily impaired in Condition White Keys compared to Condition Replicas, while humans showed no difference in their error rates between the two conditions.

The results of the supplementary test are illustrated in Figure 5. Overall, performance was better than in the main experiment, reflecting the fact that it was all assessed after the pigeons had reached criterion in the Replicas condition. However, the pigeons still varied significantly in their performance depending on Condition, $F(2,12)=31.40 ; p<.001 ; \eta_{\mathrm{p}}{ }^{2}=.84,95 \%$ CIs $=.51$, 
90. Bonferroni post-hoc tests reveal that all three conditions differed significantly from each other (all $p<.016$ ). That is, all but one pigeon performed much better under the Replicas condition than under the White Keys condition. Furthermore, all seven pigeons made more errors under the Blank condition than under the White Keys condition.

-----Figure 5 about here-----

\section{DISCUSSION}

Like the pigeons in Maes et al.'s (2015) Experiment 2A, pigeons in the main experiment acquired the task within a few sessions in Condition Replicas. In contrast, in Condition White Keys, in which a discrimination task had to be learned by pecking one of two white response keys, pigeons had still not progressed much from chance level at the end of the twenty training sessions; however, these birds progressed quickly to criterion once they had the opportunity to peck directly at a replica of the discriminative stimulus. The same difference between conditions appeared in the supplementary experiment, despite the fact that the discrimination task was arguably harder (there were two possibly relevant features rather than one) and there was some degree of overtraining (training was continued for ten sessions after criterion had been reached). Thus the stimulus-location effect is robust in pigeons.

What aspect of the learning strategy employed by pigeons led to a failure to learn the task in Condition White Keys? Learning in pigeons is typically governed by contingencies between a stimulus and its experienced consequences (Macphail, 1987; Pearce, 1987; Mackintosh, 1988; Smith et al., 2011; Smith, Berg, Cook et al., 2012). But what is the effective stimulus? The contingencies we used may have entrained considerably different conditioning processes during 
learning. Condition White Keys may have required pigeons to learn instrumentally, to move to the right or the left after seeing a certain stimulus in the display centre. Since the two response keys on the left and the right side of the stimulus were identical and presented simultaneously, the white key the pigeon was facing after orienting away from the centre key did not hold any additional information as to whether the pigeons had indeed moved towards the correct location. On the other hand, when the stimulus was replicated in the response locations, as in Condition Replicas and in Maes et al.'s Experiment 2A, a Pavlovian association becomes possible. Several accounts (Kirkpatrick-Steger \& Wasserman, 1996; Lionello \& Urcuioli, 1998; Urcuioli, 2007, 2008) have reported that the actual location in which a stimulus is presented can become integrated as a part of this stimulus. From the experimenter's perspective, the two replica images were simply the same stimulus in two different locations. For the pigeons, however, they may have constituted two distinct stimuli, with spatial location another salient feature of each stimulus.

Consider a pigeon for which horizontal orientation requires a left response, with spatial frequency irrelevant. If location becomes part of the stimulus, there are then four rewarded feature combinations (horizontal orientation, high spatial frequency with left location, horizontal orientation, low spatial frequency with left location, vertical orientation, high spatial frequency with right location, and vertical orientation, low spatial frequency with right location), and four unrewarded feature combinations (vertical orientation, high spatial frequency with left location, vertical orientation, low spatial frequency with left location, horizontal orientation, high spatial frequency with right location and horizontal orientation, low spatial frequency with right location). If the pigeons were able to treat each of these combinations as a distinct stimulus configuration in the manner envisaged by Pearce (1987), then learning the discrimination can be 
a matter of simple Pavlovian conditioning and any difficulty in discrimination will be a consequence of generalisation between the stimuli. Now contrast this with using the gratings as discriminative stimuli for a right or left response. The latter problem, requiring instrumental conditioning is likely to be more difficult to solve than the former Pavlovian discrimination, as it requires both discrimination between the stimuli and the attachment of each stimulus to the appropriate response. Consequently, generalisation can take place both between stimuli (e.g. from the horizontal orientation with low spatial frequency to the horizontal orientation with high spatial frequency) and between responses (e.g. from pecks to the white key on the left to the white key on the right). This may have contributed to the superiority of the Replica condition. Another factor may simply be that there is some intrinsic extra difficulty in learning an instrumental response for a pigeon compared to acquiring Pavlovian approach to a stimulus at a given location. Typically, both types of learning process are considered to be active during acquisition of most tasks, with some researchers going so far as to say that "every instrumental situation is a classical conditioning situation" (Sheffield, 1965, p. 317). At least for certain behaviours, classical conditioning results in better response formation than instrumental learning (Smith \& Moore, 1966, p.138). Therefore, pigeons might have been able to acquire the discrimination task easily in Condition Replicas because this condition involved a more effective classical-conditioning procedure, while pigeons in Condition White Keys had to rely almost entirely on instrumental learning.

An alternative possibility is that the geometry of the touch-screen setup - which was also used to train pigeons in Maes et al.'s experiment 2A - turned what seemed to be a simple conditioning experiment into a working memory task. Because pigeons had to stand very close to the screen to make a pecking response, they may only have had visual access to part of the 
display screen at any one time: as they moved in front of the touch screen, the stimulus and the response locations (and the images presented there) may only have appeared in their visual field one after the other rather than simultaneously. It was to test this account that we conducted the supplementary test in which, in addition to both the White Keys and Replicas conditions, pigeons also experienced Condition Blank. This was the same as the White Keys condition, except that (as in the Replicas condition) the central stimulus was removed when the response keys were presented. If, under the White Keys condition, no information was available from the central key because of the geometry of the test chamber, performance under the Blank condition should be identical to that under the White Keys condition. However, this was not the case - our results show that the pigeons did make use of the information available from the central stimulus during the time the response keys were displayed.

In contrast to the learning differences shown by pigeons, humans showed no differences in their rate of acquiring a discrimination task depending on the form of the response keys. This suggests that humans and pigeons used different strategies to acquire the discrimination task: in contrast to the contingency-dependent processes that guided pigeon behaviour, humans almost certainly used verbalised rules to acquire the discrimination task in both conditions. Two participants in Condition Replicas failed to reach the success criterion within ten blocks of training, and these were the only two people who were unable to name the underlying discrimination rule at the end of the test session. In other words, these two participants not only failed to infer the rule, but they also seemed unable to acquire the task via contingency-based learning. This is not entirely surprising given that they experienced at maximum 320 trials, whereas the pigeons (which presumably learned on the basis of contingencies alone) were allowed up to 1460 trials. 
Although our results cannot prove that the approach of testing verbalised hypotheses entirely overrides contingency learning (Doll, Jacobs, Sanfey, \& Frank, 2009) or whether, as suggested by Hayes and colleagues (1986a; 1986b; 1989), rule-governed learning impairs a subject's sensitivity to contingencies, it is apparent that knowledge of the task rule was a requirement for humans to succeed within the time we allowed them. Interestingly, the participants who successfully inferred the relevant orientation-discrimination rule (having to discriminate horizontal from vertical patterns) persistently made more errors in Condition Replicas than in Condition White Keys. Learning the spatial-frequency discrimination was not affected in any such way. Perhaps the orientation task was more cognitively demanding, and interfered with the participants' ability to apply the task rule sufficiently in Condition Replicas. Previous research (Galizio, 1979; Hayes, 1989; Maddox \& Ing, 2005; Doll et al., 2009) supports this assumption: although learning shaped by contingencies may improve performance, human behaviour is biased to be controlled by rules, even when this might be suboptimal. In the present case, simply learning the correct behaviour in response to each of the eight experienced stimuli might have resulted in more accurate performance than the rule-based approach.

The effects of task difficulty described above were only found in Condition Replicas. If human behaviour had been governed by the same learning approach in both conditions, we would have expected to observe similar effects on performance in Condition Replicas and Condition White Keys. However, both discrimination tasks were acquired equally fast in the latter condition. Furthermore, all participants in Condition White Keys were able to report the relevant discrimination rule at the end of the experiment, while two people in Condition Replicas failed to infer the task rule. Thus, we might conclude that Condition White Keys facilitates rule 
deduction in some way, making even the apparently more difficult orientation-discrimination rule obvious to participants at an earlier point.

Alternatively, the way a stimulus is presented in Condition Replicas might make the deduction of the correct discrimination rule more difficult, to the point at which it even prevents some participants from inferring the rule within the duration of the experiment. Indeed, many participants in Condition Replicas reported, when questioned about their strategy for solving the task at the end of the experiment, that the presence of two stimulus replicas in the response display confused them, and many tried to detect slight differences, for example in brightness or hue, in the two identical stimulus copies, hoping that such a perceived difference might help them choose the correct response. Obviously, this search strategy could not succeed - and participants would continue to be unable to solve the task unless they changed their strategy either by considering alternative hypotheses about the discrimination rule, or by switching to a contingency-based learning approach. Levine (1971) discovered that it can be difficult, if not impossible, for humans to discover even an easy sorting rule when it is very different from the kind of hypotheses that have worked previously to infer a rule. Thus, the participants in Condition Replicas who started out searching for differences between the two stimulus replicas might have been unable to abandon this unsuccessful hypothesis about the correct discrimination rule in favour of a hypothesis that accepts the stimulus replicas as being identical. If this is the case, then the only option that remained to enable those participants to solve the task correctly was to allow learning to be shaped not only by rules but also by the observed contingencies between the display and the correct response. Comparable conclusions have been drawn previously, e.g. by Hayes et al. (1986a), who argued that the assumed bias towards rule-governed behaviour in humans (Maddox \& Ashby, 2004; Maddox \& Ing, 2005; Smith, Beran, Crossley, 
Boomer, \& Ashby, 2010) can possibly be overcome more easily than previously thought: human performance may be shaped by an interaction between contingency-shaped and rule-governed behaviour, especially when the rules originally governing behaviour were unreliable or incomplete, as might be the case with self-generated rules.

Taken together, the pattern of human behaviour suggests that participants in Condition White Keys were able to learn a discrimination task quickly by deducing and applying the relevant discrimination rule, regardless of the potential difficulty of the task. Participants in Condition Replicas may initially have failed to deduce the appropriate task rule, which led to higher errors in responding than in Condition White Keys, especially in the more difficult orientation-discrimination task. Ultimately, this failure to learn via a rule-governed approach might have caused a shift in control of behaviour from rules to contingencies - learning the discrimination based on contingencies may then have helped to infer the correct task rule later on in the experiment, so that all successful participants were able to report the discrimination rule at the end of the experiment.

Although the procedures used with humans were modelled on those used with pigeons, some methodological differences remained, and these may allow for alternative explanations for the differences in performance of the two species.

For example, the human version of the paradigm only permitted one response before feedback was given, while pigeons were allowed to peck multiple times until the correct response key was chosen. This setup might potentially have encouraged pigeons to randomly alternate between the two keys until a reward is obtained, while humans had to perform accurately on their first try, affording a less error-prone strategy such as the deduction of rules. 
Firstly, it is indeed true that random alternations between keys would give pigeons a reward eventually, and doing so would have been less cognitively demanding than learning the discrimination task that required attention to the presented stimuli. To examine the possibility that pigeons randomly altered between the two response keys, we divided the number of pecks made to the wrong key by the number of pecks made to the correct key to calculate a pigeon's pecking bias. If a pigeon indeed alternated between keys until a reward was obtained, the pecking bias should be 1; if it preferred the correct key, this bias should be below 1 , and above 2 (since a single correct peck was required to end a trial) if the pigeon showed a bias to peck the incorrect key. These analyses reveal that pigeons in Condition Replicas soon developed a preference for pecking the correct key, with an average pecking bias declining from 3.8 in the first session to 0.2 by the time the last pigeons reached criterion in session 13 . Pigeons in Condition White Keys on the other hand developed a strong preference for pecking the incorrect key, with a pecking bias steadily inclining from 2.7 in the first session to 3.4 (i.e., pecking the wrong key 3 to 4 times before moving to the correct key) in the $20^{\text {th }}$ and final session before changing conditions. After changing to Condition Replicas, the average pecking bias dropped rapidly from 2.8 in the first session to 0.8 by the time the last pigeon reached criterion in session 16. In our view, this further supports our notion that there are different learning processes involved in the two conditions; the learning mechanisms involved in Condition White Keys enabled a more efficient stimulus control over the pigeons' behaviour than those processes required to acquire the task in Condition White Keys.

Secondly, whether or not human participants were able to correct their choice is unlikely to be the main factor determining whether or not they adopted a rule-based or contingencyshaped learning strategy. If anything, we would expect the more direct correlation between a 
response and feedback to facilitate contingency awareness above a strategy of sequentially testing the hypothetical influence of individual features of the stimuli.

Another issue that deserves attention is that the pigeons were much closer to the stimuli than the human participants. Because of the different characteristics of the human and pigeon visual systems, this difference could not be overcome. In principle, this consideration applies to many published papers in which human and pigeon performance is compared. However, although ad-hoc arguments could no doubt be constructed, it is not obvious why it would produce the effect we and previous authors have observed, other than through the memory effect which our supplementary test enabled us to discount.

\section{CONCLUSION}

Pigeons acquired a discrimination task more easily in Condition Replicas compared to Condition White Keys. Although other explanations are possible, in our view, this difference in task acquisition is convincingly explained by different conditioning processes: pigeons learned most easily via classical conditioning and failed when forced to learn in an instrumental manner. Humans on the other hand acquired a task equally well in both conditions, with any slight differences favouring Condition White Keys. They relied on knowledge of explicit task rules and failed to acquire the discrimination task if they did not have access to such rules, even if learning shaped by the contingencies of the reinforcement schedule could have led to successful task acquisition. Nonetheless, rule detection might have been facilitated by instrumental conditioning processes, especially in Condition Replicas. The differences between the two species support the dual-process theory of two separate learning systems (McLaren, Forrest, McLaren et al., 2014; Smith, Boomer, Zakrzewski et al., 2014): pigeons learned via an associative system by detecting 
contingencies between the presentation of a visual stimulus in a certain location on screen and the occurrence of food reinforcement; humans' behaviour was primarily governed by the deduction and application of task rules (though humans might be able to employ contingencybased learning methods under certain circumstances). 


\section{REFERENCES}

Beecher, M. D., \& Harrison, J. M. (1971). Rapid acquisition of an auditory localization discrimination by rats. Journal of the Experimental Analysis of Behavior, 16, 193-199.

Cardinal, R. N., \& Aitken, M. R. F. (2010). Whisker: A client-server high-performance multimedia research control system. Behavior Research Methods, 42, 1059-1071.

Doll, B. B., Jacobs, W. J., Sanfey, A. G., \& Frank, M. J. (2009). Instructional control of reinforcement learning: A behavioral and neurocomputational investigation. Brain Research, 1299, 74-94.

Erickson, M. A., \& Kruschke, J. K. (1998). Rules and exemplars in category learning. Journal of Experimental Psychology: General, 127, 107-140.

Galizio, M. (1979). Contingency-shaped and rule-governed behavior: Instructional control of human loss avoidance. Journal of the Experimental Analysis of Behavior, 31, 53-70.

Goeters, S., Blakely, E., \& Poling, A. (1992). The differential outcomes effect. The Psychological Record, 42, 389-411.

Hayes, S. C. (1989). Rule-governed behavior: Cognition, contingencies, and instructional control: Plenum Press.

Hayes, S. C., Brownstein, A. J., Haas, J. R., \& Greenway, D. E. (1986a). Instructions, multiple schedules, and extinction: Distinguishing rule-governed from schedule-controlled behavior. Journal of the Experimental Analysis of Behavior, 46, 137-147.

Hayes, S. C., Brownstein, A. J., Zettle, R. D., Rosenfarb, I., \& Korn, Z. (1986b). Rule-governed behavior and sensitivity to changing consequences of responding. Journal of the Experimental Analysis of Behavior, 45, 237-256. 
Katz, J. S., Bodily, K. D., \& Wright, A. A. (2008). Learning strategies in matching to sample: Ifthen and configural learning by pigeons. Behavioural processes, 77, 223-230.

Kirkpatrick-Steger, K., \& Wasserman, E. A. (1996). What and the where of the pigeon's processing of complex visual stimuli. Journal of Experimental Psychology: Animal Behavior Processes, 22, 60-67.

Kleiner, M., Brainard, D., \& Pelli, D. (2007). What's new in psychtoolbox-3? Paper presented at the 30th European Conference on Visual Perception, Arezzo, Italy.

Kundey, S. M. A., \& Fountain, S. B. (2014). Rats abstract rules from a response series lacking a consistent motor pattern. Learning and Motivation, 46, 44-59.

Levine, M. (1971). Hypothesis theory and nonlearning despite ideal s-r reinforcement contingencies. Psychological Review, 78, 130-140.

Lionello, K. M., \& Urcuioli, P. J. (1998). Control by sample location in pigeons' matching to sample. Journal of the Experimental Analysis of Behavior, 70, 235-251.

Mackintosh, N. J. (1988). Approaches to the study of animal intelligence. British Journal of Psychology, 79, 509-526.

Macphail, E. M. (1987). The comparative psychology of intelligence. Behavioral and Brain Sciences, 10, 645-656.

Maddox, W. T., \& Ashby, F. G. (2004). Dissociating explicit and procedural-learning based systems of perceptual category learning. Behavioural Processes, 66, 309-332.

Maddox, W. T., \& Ing, A. D. (2005). Delayed feedback disrupts the procedural-learning system but not the hypothesis-testing system in perceptual category learning. Journal of Experimental Psychology: Learning, Memory, and Cognition, 31, 100-107. 
Maes, E., De Filippo, G., Inkster, A., Lea, S. E. G., De Houwer, J., D’Hooge, R., Beckers, T., \& Wills, A. J. (2015). Feature- versus rule-based generalization in rats, pigeons and humans. Animal Cognition, 18, 1267-1284.

McLaren, I. P. L., Forrest, C. L. D., McLaren, R. P., Jones, F. W., Aitken, M. R. F., \& Mackintosh, N. J. (2014). Associations and propositions: The case for a dual-process account of learning in humans. Neurobiology of Learning and Memory, 108, 185-195.

Neill, J. C., \& Harrison, J. M. (1987). Auditory discrimination: The konorski quality-location effect. Journal of the Experimental Analysis of Behavior, 48, 81-95.

Pearce, J. M. (1987). A model for stimulus generalization in pavlovian conditioning. Psychological Review, 94, 61-73.

Peterson, G. B., \& Trapold, M. A. (1980). Effects of altering outcome expectancies on pigeons' delayed conditional discrimination performance. Learning and Motivation, 11, 267-288.

Pothos, E. M. (2005). The rules versus similarity distinction. Behavioral and Brain Sciences, 28, $1-14$.

Shanks, D. R., \& Darby, R. J. (1998). Feature-and rule-based generalization in human associative learning. Journal of Experimental Psychology: Animal Behavior Processes, 24,405 .

Sheffield, F. D. (1965). Relation between classical conditioning and instrumental learning. In W. F. Prokasy (Ed.), Classical conditioning: A symposium. New York: Appleton-CenturyCrofts.

Sheldon, M. H. (1967). Some effect of discriminable goal-box conditions on the learning of a successive discrimination. The Quarterly Journal of Experimental Psychology, 19, 319326. 
Skinner, B. F. (1969). Contingencies of reinforcement: A theoretical analysis. New York: Appleton-Century-Crofts.

Smith, J. D., Ashby, F. G., Berg, M. E., Murphy, M. S., Spiering, B., Cook, R. G., \& Grace, R. C. (2011). Pigeons' categorization may be exclusively nonanalytic. Psychonomic Bulletin \& Review, $18,414-421$.

Smith, J. D., Beran, M. J., Crossley, M. J., Boomer, J., \& Ashby, F. G. (2010). Implicit and explicit category learning by macaques (macaca mulatta) and humans (homo sapiens). Journal of Experimental Psychology: Animal Behavior Processes, 36, 54-65.

Smith, J. D., Berg, M. E., Cook, R. G., Murphy, M. S., Crossley, M. J., Boomer, J., Spiering, B., Beran, M. J., Church, B. A., Ashby, F. G., \& Grace, R. C. (2012). Implicit and explicit categorization: A tale of four species. Neuroscience \& Biobehavioral Reviews, 36, 23552369.

Smith, J. D., Boomer, J., Zakrzewski, A. C., Roeder, J. L., Church, B. A., \& Ashby, F. G. (2014). Deferred feedback sharply dissociates implicit and explicit category learning. Psychological Science, 25, 447-457.

Smith, W. I., \& Moore, J. W. (1966). Conditioning and instrumental learning. New York: McGraw-Hill.

Urcuioli, P. J. (2007). Sample and comparison location as factors in matching acquisition, transfer, and acquired equivalence. Learning \& Behavior, 35, 252-261.

Urcuioli, P. J. (2008). Associative symmetry, antisymmetry, and a theory of pigeons' equivalence-class formation. Journal of the Experimental Analysis of Behavior, 90, 257282. 
Wills, A. J., Graham, S., Koh, Z., McLaren, I. P., \& Rolland, M. D. (2011). Effects of concurrent load on feature-and rule-based generalization in human contingency learning. Journal of Experimental Psychology: Animal Behavior Processes, 37, 308.

Wills, A. J., Lea, S. E. G., Leaver, L. A., Osthaus, B., Ryan, C. M. E., Suret, M., Bryant, C. M. L., Chapman, S. J. A., \& Millar, L. (2009). A comparative analysis of the categorization of multidimensional stimuli: I. Unidimensional classification does not necessarily imply analytic processing; evidence from pigeons (columba livia), squirrels (sciurus carolinensis) and humans (homo sapiens). Journal of Comparative Psychology, 123, 391405. 


\section{AUTHOR NOTE}

The experiments described here were conducted with the approval of the Psychology Research Ethics Committee at the University of Exeter, and in accordance with UK and EU legislation, and the guidelines of the Experimental Psychology Society, for the protection of animals used in scientific experiments, and the British Psychological Society's ethical guidelines for research with human participants. We thank Guido De Filippo for discussion and Catriona Ryan for animal care and laboratory management. 


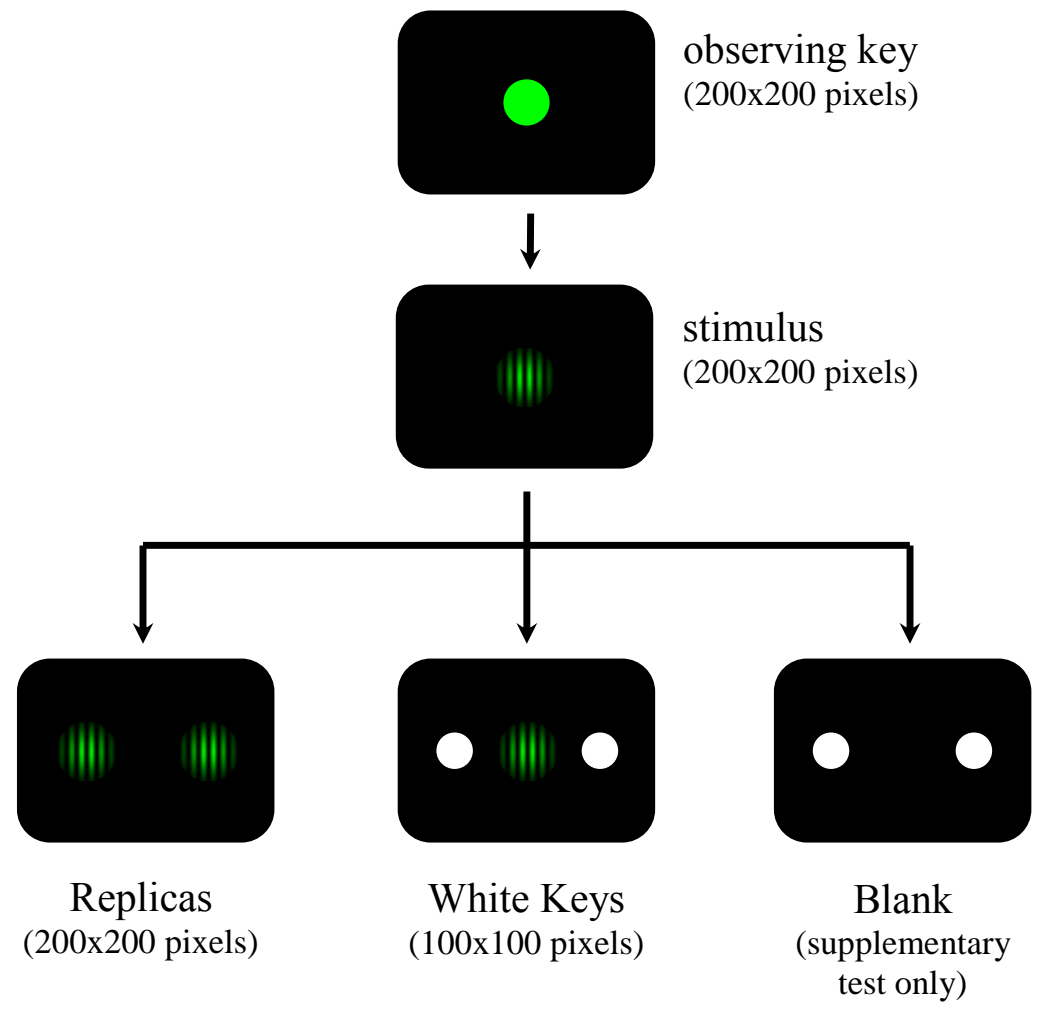

Figure 1. Example of a trial with a stimulus of vertical orientation and low spatial frequency .

Subjects first had to respond to a coloured observing key, then the stimulus appeared. After clicking or pecking the stimulus, it either disappeared from the display and reappeared to the left or the right side of the display centre (Condition Replicas), or it stayed in the display centre and two white response keys appeared on either side of it (Condition White Keys). In the supplementary test, a third condition was introduced, in which the stimulus disappeared from the display and only the two white response keys were visible (Condition Blank). A response had to made by clicking or pecking directly at a response key. 
A)

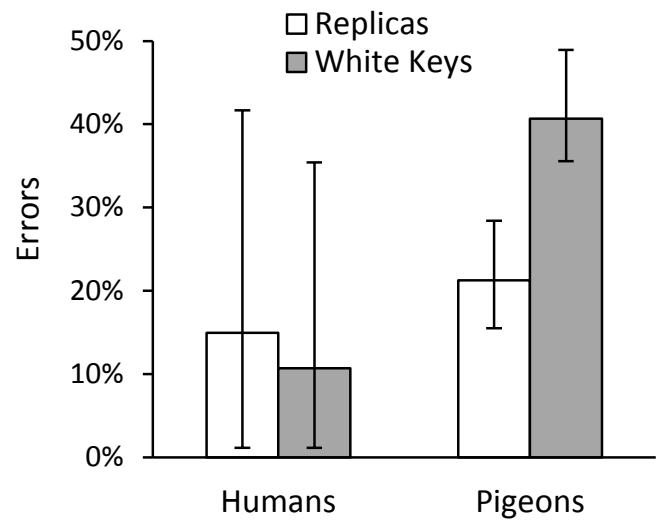

B)

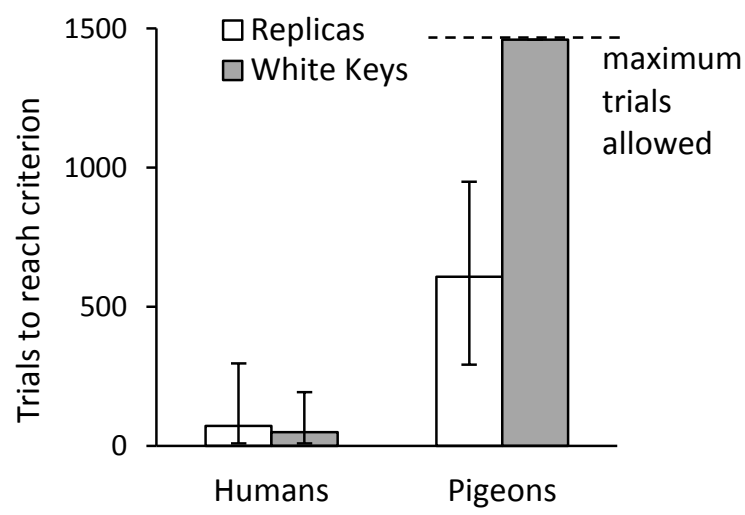

Figure 2. A) Main experiment: Error rates for humans and pigeons depending on whether subjects made a response by clicking/pecking a replica of the target stimulus (Condition Replicas) or a white response key (Condition White Keys). Error bars represent the individual range of errors.

B) Main experiment: Trials to reach the success criterion of $80 \%$ correct trials in a sequence of eight trials for humans/three consecutive training sessions for pigeons depending on condition. Error bars represent the individual range in the number of trials to reach success criterion. Note: the range for pigeons in Condition White Keys is not different from the mean. 


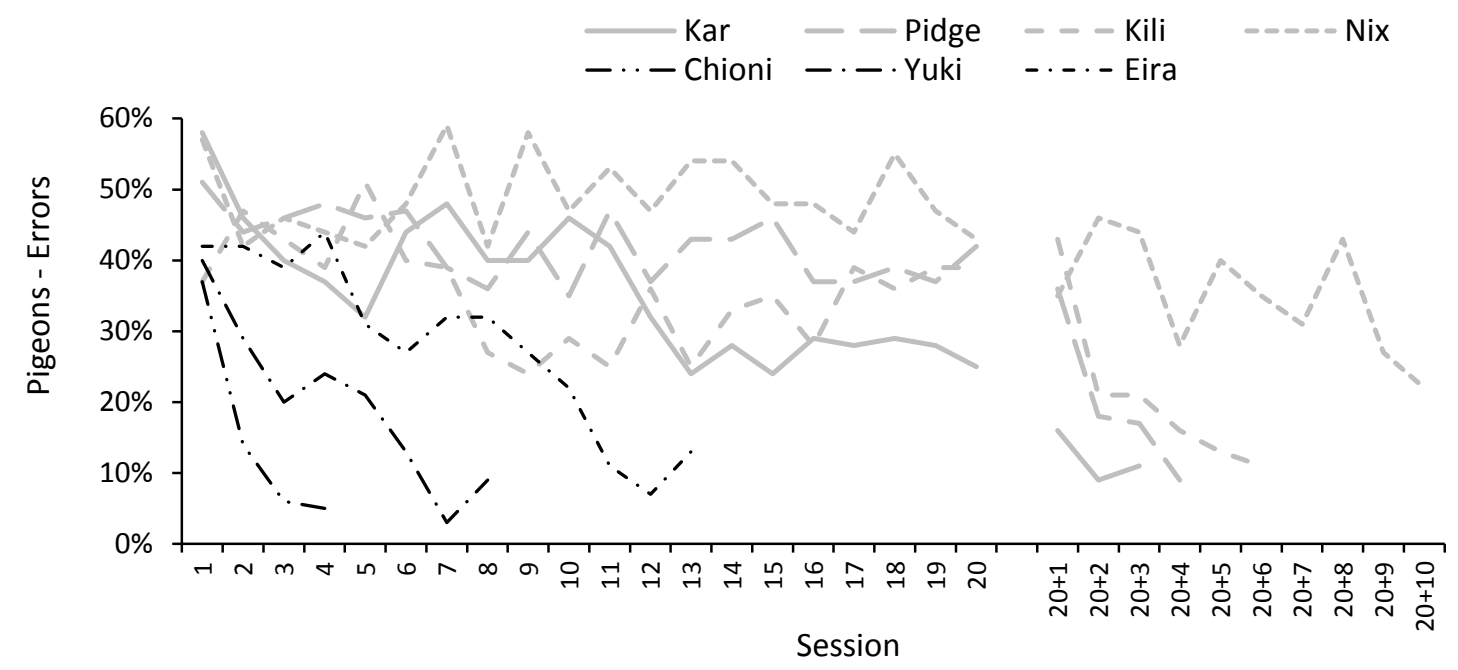

Figure 3. Main experiment: Pigeons' error rates across training sessions when making a response by pecking a replica of the target stimulus (Condition Replicas; black lines) or a white response key (Condition White Keys; grey lines). Pigeons in Condition White Keys switched to Condition Replicas after 20 sessions (performance after the switch shown as sessions $20+1$ to $20+10$ ). Kar and Chioni learned to discriminate spatial frequencies, the other pigeons performed the orientation-discrimination task. 

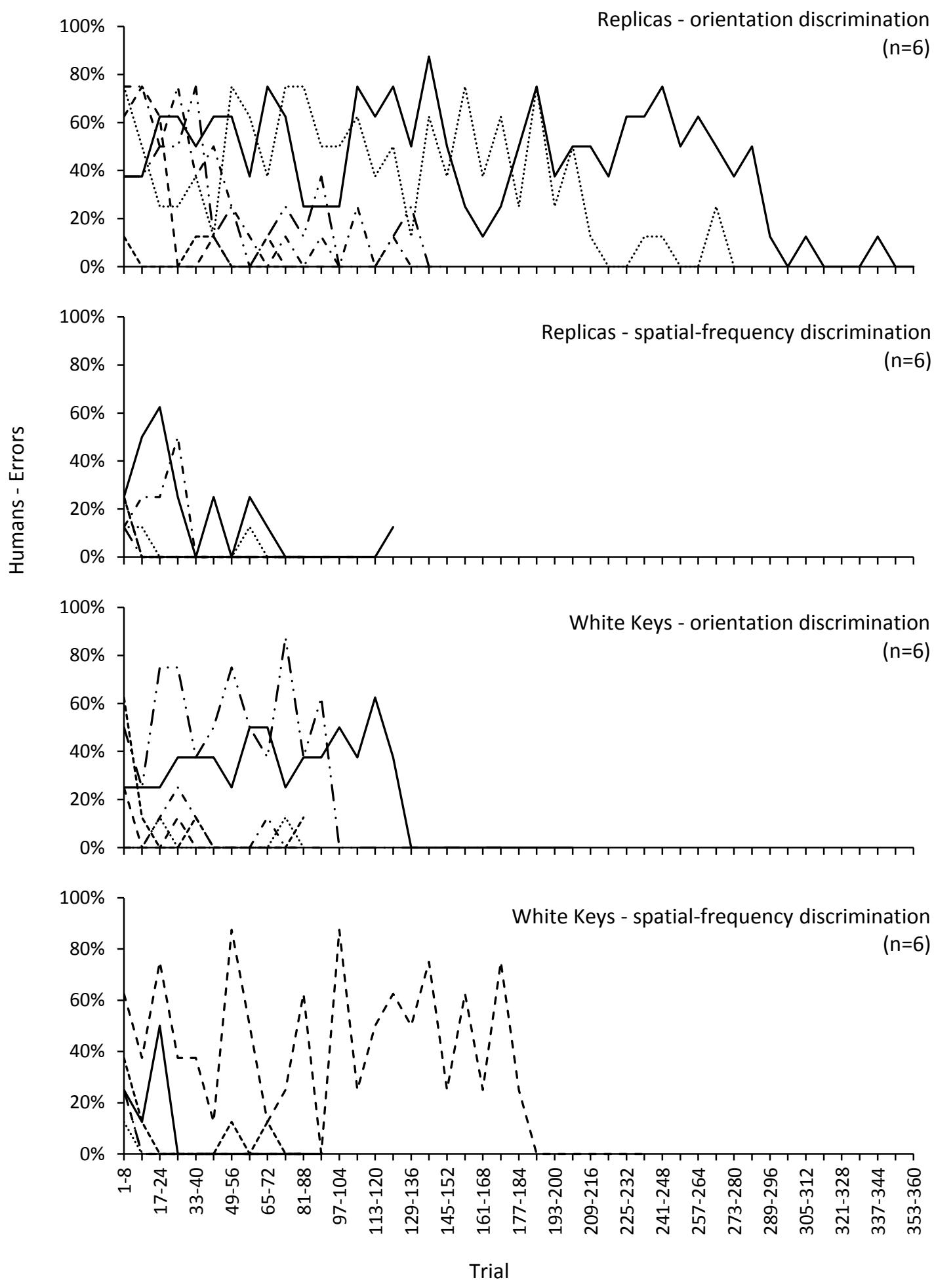

Figure 4. Main experiment: Individual human error rates depending on the condition that the participants completed (Replicas or White Keys) and the discrimination task that had to be learned (orientation discrimination or spatial-frequency discrimination). 


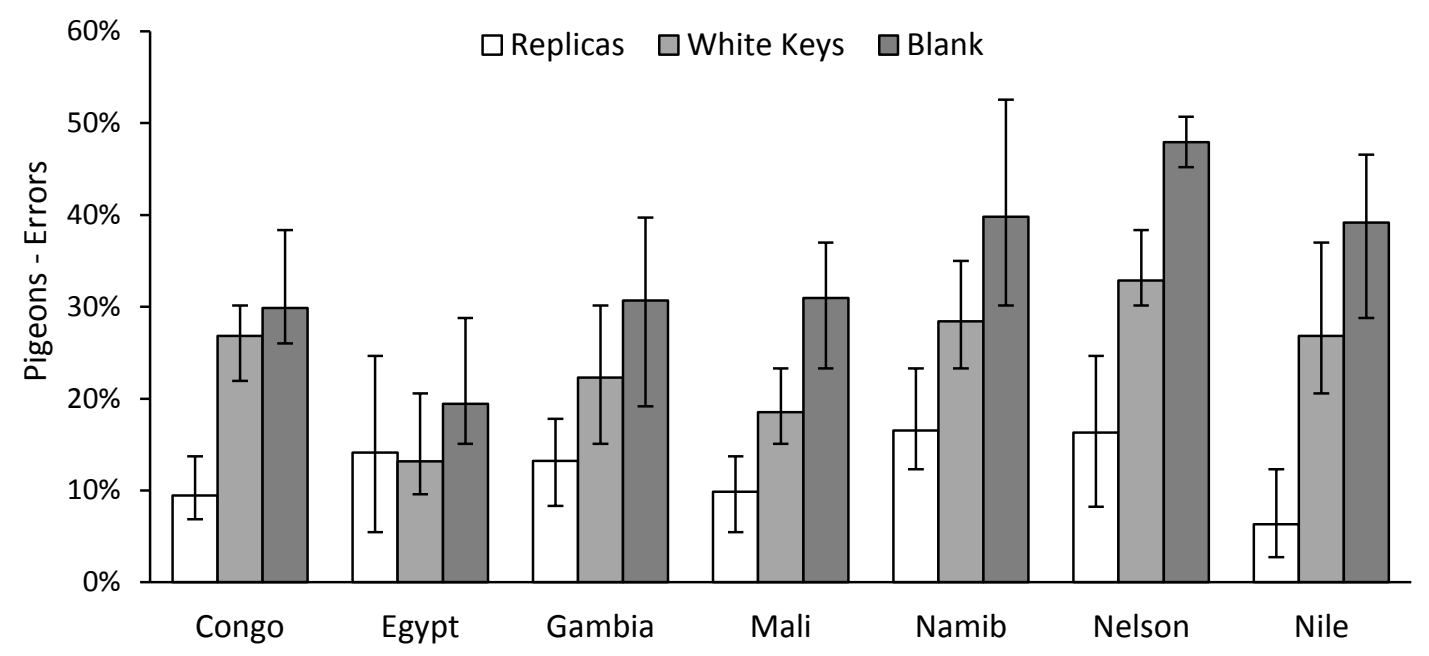

Figure 5. Supplementary test: Pigeons' error rates depending on whether subjects made a response by pecking a replica of the target stimulus (Condition Replicas) or pecking a white response key while the stimulus was visible in the centre of the screen (Condition White Keys) or while the stimulus was not presented (Condition Blank). Error bars represent the range of errors across sessions. 